\title{
VARIATION FORMULAS FOR MULTIVALENT FUNCTIONS
}

BY

\author{
A. IV. GOODMAN
}

1. Introduction. Let $R(p, q)$ be the set of functions of the form

$$
f(z)=z^{l}+\sum_{n=q+1}^{\infty} A_{n} z^{n}, \quad 1 \leqq q \leqq p,
$$

which are regular and multivalent of order $p$ in the unit circle, $|z|<1$ (hereafter denoted by $E$ ).

In recent times variation formulas for the class of normalized univalent functions $R(1,1)$ have been found, and their implications studied in some detail $[3 ; 4 ; 5 ; 12]$. However except for the work of Schiffer [12] and Gel'fer $[1 ; 2]$ nothing has been done to extend these methods to multivalent functions.

Our objective, in this work, is to examine some of the variational methods used for univalent functions, and to obtain the appropriate generalization to the class $R(p, q)$. Some of the methods which are available for the class $R(1,1)$, do not have obvious generalizations. An outstanding example of this is Loewner's method [10] which he used to prove that $\left|A_{3}\right| \leqq 3$, if $f(z) \in R(1,1)$. However in the cases which we consider here the generalizations are reasonably straightforward, and although the theorems we obtain are new, it is their various applications, which may be made in the future, which will determine their value.

A central problem in the theory of multivalent functions is that of finding sharp bounds on the coefficients $A_{n}$ in the series (1.1). Two conjectures have been advanced by the author $[6 ; 7]$ for this bound. $U_{p}$ to the present, the greatest progress in the direction of proving the first of these conjectures [6] has been made by Hayman [9], Robertson [11], and Robertson and the author [8]. With respect to the second conjecture [7], no progress has been made beyond the results proved in that paper.

2. Goluzin's method. If a function $g(z)$ is univalent in a ring $R: r \leqq|z|<1$, and is regular in $E$, then it is univalent in $E$. This almost trivial assertion becomes false if the word univalent is replaced by $p$-valent $(p \geqq 2)$, both in the hypothesis and the conclusion. This fact forces a rather complicated formulation of the main result, Theorem 2.1. To avoid this complication we introduce the following notation and definition. Let $\Delta \arg (g(z), c, \rho)$ denote the variation of $\arg \left(g\left(\rho e^{i \theta}\right)-c\right)$ for $0 \leqq \theta \leqq 2 \pi$.

Definition. A function $g(z) \not \equiv$ constant, defined in the ring $R$ is said to

Received by the editors July $11,1956$. 
satisfy the hypothesis $H(p)$ in $R$, if $p$ is the smallest integer such that $\Delta \arg (g(z), c, \rho) \leqq 2 \pi p$ for every $\rho$ in $r<\rho<1$ and for every complex $c$ such that $g\left(\rho e^{i \theta}\right) \neq c$ for real $\theta$.

Lemma 2.1. If $g(z)$ satisfies the hypothesis $H(p)$ in a ring $R$ and is regular in $E$, then it is p-valent in $E$.

The proof of this lemma is obvious.

THEOREM 2.1. Let $f(z) \in R(p, q)$ and suppose that for each real $\lambda$ in $I: 0$ $\leqq \lambda<\lambda_{0}$ the function

$$
F(z, \lambda)=f(z)+\lambda q(z)=\sum_{n=1}^{\infty} A_{n 0} z^{n}+\lambda \sum_{n=-\infty}^{+\infty} A_{n 1} z^{n}
$$

is regular and satisfies the hypothesis $H(p)$ in $R$. Suppose further that $f^{\prime}(z) \neq 0$ in $R$. Then there is a function $f^{*}(z)$ regular and p-valent in $E$ for $\lambda$ in $I_{1}: 0$ $\leqq \lambda<\lambda_{1}$ having the form

$$
\begin{aligned}
f^{*}(z) & =f(z)+\lambda\left(q(z)-z f^{\prime}(z) L(z)+z f^{\prime}(z) L_{c}(z)+O\left(\lambda^{2}\right)\right. \\
& =z^{q}\left(1+\lambda q c_{0}^{(1)}+O\left(\lambda^{2}\right)\right)+\sum_{n=q+1}^{\infty} A_{n}^{*} z^{n}
\end{aligned}
$$

where if

$$
\frac{q(z)}{z f^{\prime}(z)}=\sum_{n=-\infty}^{+\infty} c_{n}^{(1)} z^{n}
$$

then

$$
L(z)=\sum_{n=-\infty}^{-1} c_{n}^{(1)} z^{n}, \quad L_{c}(z)=\sum_{n=-\infty}^{-1} \bar{c}_{n}^{(1)} z^{-n} .
$$

Further if $F(z, \lambda)$ has no zeros in $R$ for each $\lambda$ in $I_{1}$ then $f(z)$ and $f^{*}(z)$ have the same number of zeros in $E$, when counted in accordance with their multiplicities. If $f^{\prime}(z)+\lambda q^{\prime}(z)$ has no zeros in $R$ for $\lambda$ in $I_{1}$, then when counted in accordance with their multiplicities $f(z)$ and $f^{*}(z)$ have the same number of critical points in $E$.

This theorem generalizes to $p$-valcnt functions, the one given by Goluzin $[3 ; 4 ; 5]$ for univalent functions. The proof that we present below follows closely that given by Goluzin. Some modifications have been made in an attempt to simplify the proof, and the additional hypothesis $f^{\prime}(z) \neq$ in $R$ (which is automatically satisfied for a univalent function) is necessary for the generalization. The idea behind the proof is that a substitution

$$
z=\zeta \exp \left[\sum_{\nu=1}^{\infty} \lambda^{\nu} \phi_{\nu}(\zeta)\right] \equiv h(\zeta)
$$


can be made in Equation (2.1) such that the resultant function $f^{*}(\zeta)$ is regular in $E$, while at the same time $|\zeta|=1$ is mapped by the function (2.5) univalently on $|z|=1$. It is worth noting that the possibility of such a substitution does not depend on the hypothesis that $f(z)$ is multivalent. The precise result follows.

Lemma 2.2. Let $F(z, \lambda)$ of the form (2.1) be regular in the ring $R$ for each $\lambda$ in $I$, and let $f^{\prime}(z) \neq 0$ in $R$. Then there exists a sequence of functions $\phi_{\nu}(\zeta)$, $\nu=1,2, \cdots$ such that:

(a) Each $\phi_{\nu}(\zeta)$ is regular in $R^{\prime}: r \leqq|\zeta| \leqq 1 / r$,

(b) $\Re \phi_{\nu}(\zeta)=0$ for $|\zeta|=1, \nu=1,2, \cdots$

(c) For sufficiently small $\lambda$ the series $\sum_{\nu=1}^{\infty} \lambda^{v} \phi_{\nu}(\zeta)$ is uniformly and absolutely convergent in $R^{\prime}$,

(d) The function $f^{*}(\zeta)=F(h(\zeta), \lambda)$ is regular in $E$, where $h(\zeta)$ is given by Equation (2.5).

In proving this lemma, we will need the following elementary result.

Lemma 2.3. Let

$$
g(\lambda)=\sum_{n=1}^{\infty} a_{n} \lambda^{n}
$$

where

$$
\left|a_{n}\right| \leqq 1 /(n+1)^{2}
$$$$
n=1,2, \cdots,
$$

and define $a_{n}^{(k)}$ by

$$
g^{k}(\lambda)=\sum_{n=1}^{\infty} a_{n}^{(k)} \lambda^{n}, \quad \quad k=1,2, \cdots
$$

Then there is a constant $C$ such that

$$
\left|a_{n}^{(k)}\right| \leqq \frac{C^{k}}{(n+1)^{2}}, \quad k, n=1,2, \cdots
$$

Proof. If $k=1$, then the bound (2.9) is trivial as soon as $C \geqq 1$. We proceed by induction, assuming that (2.9) has been established for all $n$, and indices $k=1,2, \cdots, K$. Then by definition

$$
a_{n}^{(K+1)}=\sum_{j=1}^{n} a_{n-j}^{(K)} a_{j},
$$

so that

$$
\left|a_{n}^{(K+1)}\right| \leqq \sum_{j=1}^{n-1} \frac{C^{K}}{(n-j+1)^{2}(i+1)^{2}}=\frac{C^{K}}{(n+1)^{2}} C^{\prime}
$$

where 


$$
C^{\prime}=\sum_{j=1}^{n-1} \frac{(n+1)^{2}}{(n-j+1)^{2}(j+1)^{2}} .
$$

But in this sum the terms for $j=k$ and $j=n-k$ are equal so that

$$
\begin{aligned}
C^{\prime} & \leqq 2 \sum_{j=1}^{[n / 2]} \frac{(n+1)^{2}}{(n-j+1)^{2}(j+1)^{2}} \leqq 2 \sum_{j=1}^{[n / 2]} \frac{1}{(j+1)^{2}} \cdot \frac{(n+1)^{2}}{(n-n / 2+1)^{2}} \\
& \leqq 8 \sum_{j=1}^{\infty} \frac{1}{(j+1)^{2}} .
\end{aligned}
$$

Therefore $C^{\prime} \leqq 8\left(\pi^{2} / 6-1\right)<6$, and it follows from inequality $(2.10)$ that the bound (2.9) is valid for all positive integers $k$ and $n$ if $C^{\prime}=6$.

To prove Lemma 2.2, we first proceed to make the substitution indicated in (d) and expand the result in a double power series in $\lambda$ and $\zeta$. The operations involved will be justified, when (c) is proved. For $k=0, \pm 1, \pm 2, \cdots$ define $B_{k n}(\zeta)$ and $Q_{k n}(\zeta)$ by

$$
\exp \left(k \sum_{\nu=1}^{\infty} \lambda^{v} \phi_{\nu}(\zeta)\right)=1+\sum_{n=1}^{\infty} B_{k n}(\zeta) \lambda^{n}=1+\sum_{n=1}^{\infty}\left(k \phi_{n}(\zeta)+Q_{k n}(\zeta)\right) \lambda^{n}
$$

where clearly $Q_{k n}(\zeta)$ depends only on $k$ and $\phi_{\nu}(\zeta), \nu=1,2, \cdots, n-1$. Using the expansion (2.11) in the series (2.1) yields

$$
\begin{aligned}
f^{*}(\zeta)= & \sum_{k=1}^{\infty} A_{k 0 \zeta^{k}}\left(1+\sum_{n=1}^{\infty}\left(k \phi_{n}(\zeta)+Q_{k n}(\zeta)\right) \lambda^{n}\right. \\
& +\lambda \sum_{k=-\infty}^{+\infty} A_{k 1} \zeta^{k}\left(1+\sum_{n=1}^{\infty} B_{k n}(\zeta) \lambda^{n}\right)
\end{aligned}
$$

or

$$
f^{*}(\zeta)=f(\zeta)+\sum_{n=1}^{\infty} \Phi_{n}(\zeta) \lambda^{n}
$$

where for $n \geqq 2$

$$
\Phi_{n}(\zeta)=\sum_{k=1}^{\infty} A_{k 0} \zeta^{k}\left(k \phi_{n}(\zeta)+Q_{k n}(\zeta)\right)+\sum_{k,=-\infty}^{+\infty} A_{k 1} \zeta^{k} B_{k, n-1}(\zeta)
$$

and

$$
\Phi_{1}(\zeta)=\sum_{k=1}^{\infty} k A_{k 0} \zeta^{k} \phi_{1}(\zeta)+\sum_{k=-\infty}^{+\infty} A_{k 1} \zeta^{k}=\phi_{1}(\zeta) \zeta f^{\prime}(\zeta)+q(\zeta) .
$$

Now for $n \geqq 2, \Phi_{n}(\zeta)$ can be written in the form

$$
\Phi_{n}(\zeta)=\phi_{n}(\zeta) \zeta f^{\prime}(\zeta)+\sum_{k=1}^{\infty} A_{k n} \zeta^{k} Q_{k n}(\zeta)+\sum_{k=-\infty}^{+\infty} A_{k 1} \zeta^{k} B_{k, n-1}(\zeta)
$$


and each term on the right side except the first involves $\phi_{\nu}(\zeta)$ only with indices $\nu<n$. Thus equation (2.14) suggests that $\phi_{n}(\zeta)$ can be defined inductively so as to make $\Phi_{n}(\zeta)$ regular in $E$. To begin the procedure with $\Phi_{1}(\zeta)$, we expand $q(\zeta) / \zeta f^{\prime}(\zeta)$ as a Laurent series in $R$, Equation (2.3), and set

$$
\phi_{1}(\zeta)=-L(\zeta)+L_{c}(\zeta)
$$

where $L(\zeta)$ is defined by Equation (2.4). It is now clear that $\phi_{1}(\zeta)$ satisfies the conditions (a) and (b) of Lemma 2.2, and with respect to (d) the function $\Phi_{1}(\zeta)$ is regular in $|\zeta|<1$, since now

$$
\Phi_{1}(\zeta)=\zeta f^{\prime}(\zeta)\left[\phi_{1}(\zeta)+\frac{q(\zeta)}{\zeta f^{\prime}(\zeta)}\right]
$$

is a product of two such functions.

Before considering the condition (c) of the lemma we introduce a convenient symbol, namely if

$$
\gamma(\zeta)=\sum_{n=-\infty}^{\infty} c_{n} \zeta^{n}
$$

then we set

$$
\|\gamma(\zeta)\|=\sum_{n=-\infty}^{+\infty}\left|c_{n} \zeta^{n}\right|
$$

If the series involved converge, it is obvious that

$$
\left\|\gamma_{1}(\zeta)+\gamma_{2}(\zeta)\right\| \leqq\left\|\gamma_{1}(\zeta)\right\|+\left\|\gamma_{2}(\zeta)\right\|
$$

and

$$
\left\|\gamma_{1}(\zeta) \cdot \gamma_{2}(\zeta)\right\| \leqq\left\|\gamma_{1}(\zeta)\right\| \cdot\left\|\gamma_{2}(\zeta)\right\| .
$$

To satisfy the condition (c) of the lemma, we will show that there are constants $D$ and $M$ such that for $\zeta$ in $R^{\prime}$

$$
\left\|\phi_{\nu}(\zeta)\right\| \leqq D M^{\nu-1} /(\nu+1)^{2}, \quad \nu=1,2, \cdots
$$

This inequality will certainly imply the absolute and uniform convergence of the series in (c) for $|\lambda| \leqq M^{-1}$. For $\nu=1$, it suffices to set $D=4\left\|\phi_{1}(r)\right\|$ and (2.17) is satisfied.

For $n \geqq 2$ we write Equation (2.14) in the form

$$
\Phi_{n}(\zeta)=\zeta f^{\prime}(\zeta)\left\{\phi_{n}(\zeta)+\tau_{n}(\zeta)\right\}
$$

where

$$
\tau_{n}(\zeta) \zeta f^{\prime}(\zeta)=\sum_{k=1}^{\infty} A_{k 0} \zeta^{k} Q_{k n}(\zeta)+\sum_{k=-\infty}^{+\infty} A_{k 1} \zeta^{k} B_{k, n-1}(\zeta)
$$


Using Equation (2.11), it is easy to see that the right side of Equation (2.19) is merely the coefficient of $\lambda^{n}$ in the expression

$$
\sum_{k=-\infty}^{+\infty} \sum_{l=0}^{1} A_{k l} \zeta^{k} \lambda^{l} \exp \left(k \sum_{\nu=1}^{n-1} \lambda^{\nu} \phi_{\nu}(\zeta)\right)
$$

where for convenience we have set $A_{k 0}=0$ if $k<1$. Then

$$
\tau_{n}(\zeta)=\frac{1}{n ! \zeta f^{\prime}(\zeta)} \frac{d^{n}}{d \lambda^{n}}\left(\sum \sum A_{k l} \zeta^{k} \lambda^{l} \exp \left(k \sum \lambda^{\nu} \phi_{\nu}(\zeta)\right)\right)_{\mid \lambda=0}
$$

where here and in the next three equations the range of summation is the same as in Equation (2.20). The properties of the symbol \|| || yield

$$
\left\|\tau_{n}(\zeta)\right\| \leqq\left.\left\|\frac{1}{\zeta f^{\prime}(\zeta)}\right\| \frac{1}{n !} \frac{d^{n}}{d \lambda^{n}}\left(\sum \sum\left|A_{k l} \zeta^{k}\right| \lambda^{l} \exp \left(|k| \sum \lambda^{\nu}\left\|\phi_{\nu}(\zeta)\right\|\right)\right)\right|_{\lambda=0} .
$$

For brevity set $B=\left\|1 / r f^{\prime}(r)\right\|$ and assume that for $|\zeta|=r$, the inequality (2.17) has been established for indices $\nu=1,2, \cdots, n-1$. Then for $|\zeta|=r$

$$
\left\|\tau_{n}(r)\right\| \leqq\left. B \frac{1}{n !} \frac{d^{n}}{d \lambda^{n}}\left(\sum \sum\left|A_{k j}\right| r^{k} \lambda^{l} \exp \left(|k| \sum \lambda^{\nu} D M^{\nu-1} /(\nu+1)^{2}\right)\right)\right|_{\lambda=0} .
$$

If we replace $\lambda$ by $\mu / M$, and consequently $d \Sigma / d \lambda$ by $M d \Sigma / d \mu$, then

$$
\left\|\tau_{n}(r)\right\| \leqq\left.\frac{B M^{n}}{n !} \frac{d^{n}}{d \mu^{n}}\left(\sum \sum\left|A_{k l}\right| \frac{r^{k} \mu^{l}}{M^{l}} \exp \left(|k| D M^{-1} \sum \mu^{\nu} /(\nu+1)^{2}\right)\right)\right|_{\mu=0},
$$

or

$$
\begin{gathered}
\left\|\tau_{n}(r)\right\| \leqq \\
\left.\quad\left(\sum_{\nu=1}^{n} \sum_{k=-\infty}^{+\infty} \frac{\mu^{\nu}}{(\nu+1)^{2}}\right)^{j}\right|_{\mu=0} \cdot A_{k l \mid} \mid r^{k} \sum_{j=0}^{\infty} \frac{|k|^{j} D^{j}}{M^{l+j} j !} \frac{1}{n !} \frac{d^{n}}{d \mu^{n}} \mu^{l}
\end{gathered}
$$

Since $n \geqq 2$, the terms for which $l+j<2$ give zero after the differentiation, hence in what follows we may assume $l+j \geqq 2$. The standard formula for the $n$th derivative of a product yields

$$
\left.\frac{1}{n !} \frac{d^{n}}{d \mu^{n}} \mu^{l}\left(\sum_{\nu=1}^{n-1} \frac{\mu^{\nu}}{(\nu+1)^{2}}\right)^{j}\right|_{\mu=0}=\left.\frac{1}{(n-l) !} \frac{d^{n-l}}{d \mu^{n-l}}\left(\sum_{\nu=1}^{n-1} \frac{\mu^{\nu}}{(\nu+1)^{2}}\right)^{j}\right|_{\mu=0} .
$$

Now apply Lemma 2.3 to the right side of this equation. Then for $n \geqq 2$

$$
\left.\frac{1}{n !} \frac{d^{n}}{d \mu^{n}} \mu^{l}\left(\sum_{\nu=1}^{n-1} \frac{\mu^{\nu}}{(\nu+1)^{2}}\right)^{i}\right|_{\mu=0} \leqq \frac{C^{j}}{(n-l+1)^{2}},
$$

and using this in Equation (2.21) gives 


$$
\left\|\tau_{n}(r)\right\| \leqq B M^{n} \sum_{k, l, j}\left|A_{k l}\right| \frac{r^{k}}{M^{l+j}} \frac{|k|^{j} D^{j} C^{j}}{j !(n-l+1)^{2}} .
$$

But $l=0,1$, and $n \geqq 2$, so $(n+1)^{2} /(n-l+1)^{2}<3$. Thus the inequality (2.24) becomes

$$
\left\|\tau_{n}(r)\right\| \leqq \frac{3 B M^{n}}{(n+1)^{2}} \sum_{k, l}\left|A_{k l}\right| \frac{r^{k}}{M^{l}} e^{|k| D C / M}=\frac{3 B M^{n}}{(n+1)^{2}} S .
$$

We first observe that for large $M$ the series for $S$ is convergent, since $f(z, \lambda)$ is regular in $R$ for each $\lambda$ in $I$. But then from Equation (2.24) $S=O\left(M^{-2}\right)$ since $l+j \geqq 2$. Finally we note that the sum $S$ is independent of $n$, so that if $M$ is taken sufficiently large in the inequality (2.17) and if this bound is assumed valid for $\nu=1,2, \cdots, n-1$, then

$$
\left\|\tau_{n}(r)\right\| \leqq \frac{3 B M^{n}}{(n+1)^{2}} \cdot \frac{C^{\prime \prime}}{M^{2}} \leqq \frac{D M^{n-1}}{2(n+1)^{2}} .
$$

Now set

$$
\tau_{n}(\zeta)=\sum_{k=-\infty}^{+\infty} c_{k}^{(n)} \zeta^{k}
$$

Then the bound (2.26) implies that

$$
\sum_{k=-\infty}^{-1}\left|c_{k}^{(n)}\right| r^{k} \leqq \frac{D M^{n-1}}{2(n+1)^{2}}
$$

so that if we define $\phi_{n}(\zeta)$ by

$$
\phi_{n}(\zeta)=-\sum_{k=-\infty}^{-1} c_{k}^{(n)} \zeta^{k}+\sum_{k=-\infty}^{-1} \bar{c}_{k}^{(n)} \zeta^{-k}
$$

then $\phi_{n}(\zeta)$ satisfies the inequality (2.17) for $|\zeta|=r$, and consequently for $\zeta$ in $R^{\prime}$. If we take $0 \leqq \lambda \leqq 1 / M$, then the series in part (c) of Lemma 2.1 will be uniformly and absolutely convergent for $\zeta$ in $R^{\prime}$. Further with this definition of $\phi_{n}(\zeta), \Re \phi_{n}\left(e^{i \theta}\right)=0$.

Finally we mention that the series (2.1) is absolutely convergent for $r \leqq|\zeta| \leqq r_{1}<1$ so that all of the formal operations used are justified for $\zeta$ in this ring, and small $\lambda$. But Equations (2.18), (2.27) and (2.28) show that $\Phi_{n}(\zeta)$ is regular for $|\zeta| \leqq r_{1}$, so $f^{*}(\zeta)$ given by Equation (2.12) is regular in this circle, and consequently in $E$. This completes the proof of Lemma 2.2.

With the aid of this lemma, the proof of Theorem 2.1 is easy. Indeed the substitution $z=h(\zeta)$ dictated by Equation (2.5) is made in $F(z, \lambda)$ giving $f_{\lambda}^{*}(\zeta)=f^{*}(\zeta)$ regular in $E$. For sufficiently small $\lambda, h(\zeta)$ is regular in $R^{\prime}$ and maps $|\zeta|=1$ univalently onto $|z|=1$ preserving direction. Consequently 
there is a doubly-connected region $D$ in the $\zeta$ plane whose outer boundary is $|\zeta|=1$, in which $h(\zeta)$ is univalent and maps $D$ onto the ring $R_{1}: r_{1}<|z|<1$, $r \leqq r_{1}$. Thus $D$ is covered by the level curves $\Gamma_{\rho}$ of $h(\zeta)$ along which $|h(\zeta)|=\rho$, $r_{1}<\rho<1$. But $f^{*}(\zeta)$ maps $\Gamma_{\rho}$ onto the same curve that $F(z, \lambda)$ maps $|z|=\rho$. Then by the hypothesis $H(p), \Delta \arg \left(f^{*}(\zeta)-c\right) \leqq 2 \pi p$ on $\Gamma_{\rho}$, as long as $f^{*}(\zeta) \neq c$ on $\Gamma_{\rho}$. Thus $f^{*}(\zeta)$ is $p$-valent in $E$.

To see that $f^{*}(\zeta)$ has the form of Equation (2.2), with $z$ replaced by $\zeta$, it is sufficient to observe that the coefficient of $\lambda$ is $\Phi_{1}(\zeta)$ and this is determined by the Equations (2.16), (2.15), (2.3) and (2.4). Further each $\Phi_{n}(\zeta)$ is the product of $\zeta f^{\prime}(\zeta)$ and a function regular in $E$, so $f^{*}(\zeta)$ has a $q$ th order zero at the origin.

Let $s$ and $t$ denote the number of zeros and critical points respectively of $f(z)$ in $E$. By hypothesis both $s$ and $t$ are finite, and all of the zeros and critical points lie in $E-R$. Since $F(z, \lambda)$ has no zeros in $R$, for $\lambda$ in $I$

$$
\int_{|z|=\rho_{1}} \frac{F^{\prime}(z, \lambda)}{F(z, \lambda)} d z=\int_{|z|=\rho_{2}} \frac{F^{\prime}(z, \lambda)}{F(z, \lambda)} d z \equiv J
$$

where $r_{1}<\rho_{1}<\rho_{2}<1$, and both integrals are in the counterclockwise direction. But the right side of Equation (2.29) is an integer multiple of $2 \pi i$, and the integrand is continuous in $\lambda$, so that on letting $\lambda \rightarrow 0$, we find $J=2 \pi i s$. But the first integral in Equation (2.29) is

$$
\int_{\Gamma_{\rho_{1}}} \frac{f^{* \prime}(\zeta)}{f^{*}(\zeta)} d \zeta
$$

since both integrals are $\Delta \arg w$, on a curve which is simultaneously the image of $|z|=\rho_{1}$ under $f(z)$, and the image of $\Gamma_{\rho_{1}}$ under $f^{*}(\zeta)$. Therefore $f^{*}(\zeta)$ has $s$ zeros in the region enclosed by $\Gamma_{\rho_{1}}$, and consequently $s$ zeros in $E$.

The same procedure can be used to show that

$$
\int_{\Gamma_{\rho_{1}}} \frac{F^{\prime \prime}(h(\zeta), \lambda)}{F^{\prime}(h(\zeta), \lambda)} h^{\prime}(\zeta) d \zeta=2 \pi i t
$$

if $F^{\prime}(z, \lambda)$ has no zeros in $R$ for $\lambda$ in $I$. In terms of $f^{*}(\zeta)$, this yields

$$
\int_{\Gamma_{\rho_{1}}}\left(\frac{f^{* \prime \prime}(\zeta)}{f^{* \prime}(\zeta)}-\frac{h^{\prime \prime}(\zeta)}{h^{\prime}(\zeta)}\right) d \zeta=2 \pi i t
$$

But $h^{\prime}(\zeta) \rightarrow 1$ uniformly in $R_{1}$ as $\lambda \rightarrow 0$, so for $\lambda$ small, $\Delta$ arg $h^{\prime}(\zeta)=0$, thus $f^{*}(\zeta)$ has $t$ critical points in $E$. This completes the proof of Theorem 2.1.

To apply this theorem we must make a suitable choice of $q(z)$. The following lemma will be useful in constructing $q(z)$.

Lemma 2.4. Let $b_{k}, B_{k}(k=1,2, \cdots, m)$ be finite complex constants, and let $P$ denote the closed region obtained by deleting from the extended complex 
plane the $m$ mutually disjoint disks $\left|w-b_{k}\right|<\epsilon(\epsilon>0)$. Then there is $a \lambda(\epsilon)>0$ such that for each $\lambda$ in $0 \leqq \lambda<\lambda(\epsilon)$ the function

$$
\phi(w)=w+\lambda \sum_{k=1}^{m} \frac{B_{k} w^{2}}{w-b_{k}}
$$

is univalent in $P$, preserves the point at infinity, and maps the boundary $\left|w-b_{k}\right|$ $=\epsilon$ of each disk onto a simple closed convex curve. Further $\phi^{\prime}(w)$ is not zero in $P$.

The fact that $\phi(w)$ is univalent in $P$ for $0 \leqq \lambda<\lambda_{0}$ follows immediately from the identity

$$
\frac{\phi\left(w_{1}\right)-\phi\left(w_{2}\right)}{w_{1}-w_{2}}=1+\lambda \sum_{k=1}^{m} B_{k} \frac{w_{1} w_{2}+b_{k}\left(w_{1}+w_{2}\right)}{\left(w_{1}-b_{k}\right)\left(w_{2}-b_{k}\right)},
$$

since the sum is uniformly bounded in $P$ and hence the right side cannot vanish if $\lambda$ is sufficiently small.

A necessary and sufficient condition for $\phi(w)$ to map $\left|w-b_{j}\right|=\epsilon$ onto a strictly convex curve is that

$$
1+\Re\left(w-b_{j}\right) \phi^{\prime \prime}(w) / \phi^{\prime}(w)>0
$$

for $w=b_{j}+\epsilon e^{i \theta}, 0 \leqq \theta \leqq 2 \pi$. Applied to $\phi(w)$ defined by Equation (2.30) this yields

$$
1+\lambda \Re\left(w-b_{j}\right) \frac{\sum_{k=1}^{m} 2 B_{k} b_{k}^{2}\left(w-b_{k}\right)^{-3}}{1+\lambda \sum_{k=1}^{m} B_{k}\left(1-b_{k}\left(w-b_{k}\right)^{-2}\right)}>0,
$$

and it is obvious that for each index $j$, there is a positive $\lambda_{j}$ such that the inequality (2.32) is satisfied for $w=b_{j}+\epsilon e^{i \theta}$ and $0 \leqq \lambda<\lambda_{j}$. It only remains to set $\lambda(\epsilon)=\min \left\{\lambda_{0}, \lambda_{1}, \cdots, \lambda_{m}\right\}$. Finally since $\phi(w)$ is univalent in $P$, the image curves of $\left|w-b_{j}\right|=\epsilon$ are simple curves, and further $\phi^{\prime}(w) \neq 0$ in $P$.

Because of the convexity property just proved, it is easy to extend the mapping of $P$ defined by Equation (2.30) to a mapping that is continuous and univalent in the entire complex plane (although the extended function need not be analytic in the circles $\left.\left|w-b_{j}\right| \leqq \epsilon\right)$. We consider this extension made and suppose $\Gamma$ is any closed curve in the $w$-plane with only a finite number of self-intersection points, and suppose $c$ is any point not on $\Gamma$. Let $\Gamma^{*}$ and $c^{*}$ be the image curve and point under $w^{*}=\phi(w)$. Since $\phi(w)$ is now univalent, continuous in the extended plane and preserves the point at infinity,

$$
\Delta_{\Gamma} \arg (w-c)=\Delta_{\Gamma *} \arg \left(w^{*}-c^{*}\right) .
$$

With these preparations we can easily prove 
THEOREM 2.2. Let $f(z) \in R(p, q)$ and let $f^{\prime}(z)$ have no zeros in $R: r \leqq|z|<1$. Let $b_{k}, k=1,2, \cdots, m$ be $m$ distinct points, each at positive distance from $V$, the boundary $\left(^{1}\right)$ of the image of $E$ under $f(z)$. Then the function

$$
F(z, \lambda)=f(z)+\lambda \sum_{k=1}^{m} \frac{B_{k} f^{2}(z)}{f(z)-b_{k}}
$$

satisfies the hypotheses of Theorem 2.1. Thus

$$
q(z)=\sum_{k=1}^{m} \frac{B_{k} f^{2}(z)}{f(z)-b_{k}},
$$

and $f^{*}(z)$ given by Equation (2.2) is p-valent in E. Further $f(z)$ and $f^{*}(z)$ have in $E$, the same number of zeros and the same number of critical points, when counted in accordance with their multiplicities.

Proof. Since each $b_{k}$ is a positive distance from $V$, there is an $\epsilon>0$ such that all points of the circles $\left|w-b_{k}\right| \leqq \epsilon, k=1,2, \cdots, m$ have this same property. Then there is a ring $R_{1}: r_{1} \leqq|z|<1$ such that its image under $f(z)$ does not meet these circles. But $f(z)$ is $p$-valent in $E$ and hence in $R_{1}$ so $f(z)$ satisfies the hypothesis $H(p)$ in this ring. On the other hand the function $\phi(w)$ is univalent in $P$ for sufficiently small $\lambda$ so that if $w$ is replaced by $f(z)$ in Equation (2.30) then the resulting function $F(z, \lambda)$, Equation (2.34) also satisfies the hypothesis $H(p)$. For if $\Gamma$ is the image of $|z|=\rho, r_{1}<\rho<1$ under $f(z)$, then $\Gamma^{*}$ is the image of the same circle under $F(z, \lambda)$ and our assertion follows from Equation (2.33).

Further it is obvious that for sufficiently small $\lambda, F(z, \lambda) \neq 0$ in $R_{1}$. The same is true of the derivative, since

$$
d F / d z=\phi^{\prime}(w) f^{\prime}(z)
$$

$\phi^{\prime}(w) \neq 0$ in $P$, and $f^{\prime}(z) \neq 0$ in $R$.

To apply this theorem we must compute $L(z)$ for $q(z) / z f^{\prime}(z)$ when $q(z)$ has the form (2.35). The poles of this function consist of the critical points of $f(z)$ and the zeros of $f(z)-b_{k}$. For simplicity we assume that $m=1$, and set $b_{1}=b \neq 0, B_{1}=B \neq 0$. We assume further that the roots $\alpha_{k}$ of $f(z)-b=0$ are simple. Then the residue at any one of the roots $z=\alpha$, is

$$
\lim _{z \rightarrow \alpha}(z-\alpha) \frac{1}{z f^{\prime}(z)} \frac{B f^{2}(z)}{f(z)-f(\alpha)}=\frac{B}{\alpha}\left(\frac{f(\alpha)}{f^{\prime}(\alpha)}\right)^{2}
$$

and the contribution to $L(z)$ is $B L_{1}(\alpha) /(z-\alpha)$ where

$$
L_{1}(\alpha)=\frac{1}{\alpha}\left(\frac{f(\alpha)}{f^{\prime}(\alpha)}\right)^{2} .
$$

(1) By the boundary of the image of $E$, we understand the collection of all limit points of all sequences $\left\{f\left(z_{n}\right)\right\}$ as $\left|z_{n}\right| \rightarrow 1$ with $\left|z_{n}\right|<1$. 
In a like manner if $\beta \neq 0$ is a simple zero of $f^{\prime}(z)$ then the contribution to $L(z)$ is $B L_{2}(\beta) /(z-\beta)$, where

$$
L_{2}(\beta)=\frac{f^{2}(\beta)}{\beta(f(\beta)-b) f^{\prime \prime}(\beta)} .
$$

If $f^{\prime}(z)$ has multiple zeros then the contribution is more complicated. Since $f(0)=0$, it is clear that $q(z) / z f^{\prime}(z)$ is regular at $z=0$. This gives the following

Theorem 2.3. Let $f(z) \in R(p, q), q \geqq 1$, and suppose that in $E, f^{\prime}(z)$ has only a finite number of zeros $\beta_{k}$, all simple, $0<\left|\beta_{k}\right|<r<1,(k=1,2, \cdots, t)$. Let $V$ be the boundary of the image of $E$ under $f(z)$ let $b$ be a complex number $(b \neq 0, b \notin V)$ for which all the roots $\alpha_{k}(k=1,2, \cdots, s)$ of $f(z)=b$ are simple. Then $f^{*}(z)$, given by Equation (2.2), is a neighboring p-valent function, where in Equation (2.2)

$$
L(z)=B \sum_{k=1}^{s} \frac{1}{z-\alpha_{k}} L_{1}\left(\alpha_{k}\right)+B \sum_{k=1}^{t} \frac{1}{z-\beta_{k}} L_{2}\left(\beta_{k}\right),
$$

and

$$
L_{c}(z)=\bar{B} \sum_{k=1}^{s} \frac{z}{1-\bar{\alpha}_{k} z} \bar{L}_{1}\left(\alpha_{k}\right)+\bar{B} \sum_{k=1}^{t} \frac{z}{1-\bar{\beta}_{k} z} \bar{L}_{2}\left(\beta_{k}\right) .
$$

Further $f(z)$ and $f^{*}(z)$ have in $E$ the same number of zeros, and the same number of critical points when counted in accordance with their multiplicities.

If the neighboring function is to be an element of the class $R(p, q)$ we must divide by $A_{q}^{*}$, the coefficient of $z^{q}$ in $f^{*}(z)$. Although this is given by Equations (2.2) and (2.3), the computation of $A_{q}^{*}$ is done more easily by letting $z \rightarrow 0$ in $f^{*}(z) / z^{q}$, using Equations (2.38), (2.39) and (2.35) with $m=1$. This yields

$$
A_{q}^{*}=1+\lambda q B\left\{\sum_{k=1}^{8} L_{1}\left(\alpha_{k}\right) / \alpha_{k}+\sum_{k=1}^{t} L_{2}\left(\beta_{k}\right) / \beta_{k}\right\}+O\left(\lambda^{2}\right) .
$$

This gives the following result for variation in the family $R(p, q)$.

THEOREM 2.4. Under the conditions and with the notations of Theorem 2.3, $f_{\lambda}(z) \in R(p, q)$ where

$$
\begin{aligned}
f_{\lambda}(z)= & f(z)+\lambda\left\{\frac{B f^{2}(z)}{f(z)-b}-q B f(z)\left[\sum_{k=1}^{s} \frac{L_{1}\left(\alpha_{k}\right)}{\alpha_{k}}+\sum_{k=1}^{t} \frac{L_{2}\left(\beta_{k}\right)}{\beta_{k}}\right]\right. \\
& +\bar{B} z f^{\prime}(z)\left[\sum_{k=1}^{8} \frac{z}{1-\bar{\alpha}_{k} z} \bar{L}_{1}\left(\alpha_{k}\right)+\sum_{k=1}^{t} \frac{z}{1-\bar{\beta}_{k} z} \bar{L}_{2}\left(\beta_{k}\right)\right] \\
& \left.-B z f^{\prime}(z)\left[\sum_{k=1}^{s} \frac{1}{z-\alpha_{k}} L_{1}\left(\alpha_{k}\right)+\sum_{k=1}^{t} \frac{1}{z-\beta_{k}} L_{2}\left(\beta_{k}\right)\right]\right\}+O\left(\lambda^{2}\right) .
\end{aligned}
$$


Further for sufficiently small $\lambda, f(z)$ and $f_{\lambda}(z)$ have in $E$, the same number of zeros and critical points when counted in accordance with their multiplicities.

If in Equation (2.35) more than one term is used, then Equation (2.41) is modified by the addition of further terms in an obvious fashion.

If we alter $q(z)$ we can obtain an analogous result. First we state

LEMma 2.5. With the notations and conditions of Lemma 2.4 the function

$$
\psi(w)=w+\lambda \sum_{k=1}^{m} \frac{B_{k} w}{w-b_{k}}
$$

is univalent in $P$, preserves the point at infinity and maps each curve $\left|w-b_{k}\right|=\epsilon$ onto a simple closed convex curve, for $\lambda$ a sufficiently small positive number.

The proof of this lemma is similar to the proof of Lemma 2.4, so it is omitted. In the same way that Lemma 2.4 leads to Theorems 2.2, 2.3 and 2.4, Lemma 2.5 leads to analogous theorems. We omit the details, and merely give the formula for the neighboring normalized $p$-valent function. Using $b_{1}=b$, and one term in Equation (2.42), we find

$$
\begin{aligned}
f_{\lambda}(z)= & f(z)+\lambda\left\{\frac{B_{1}}{b} \frac{f^{2}(z)}{f(z)-b}-\frac{q B_{1} f(z)}{b}\left[\sum_{k=1}^{s} \frac{L_{1}\left(\alpha_{k}\right)}{\alpha_{k}}+\sum_{k=1}^{t} \frac{L_{2}\left(\beta_{k}\right) b}{\beta_{k} f\left(\beta_{k}\right)}\right]\right. \\
& +\bar{B}_{1} z f^{\prime}(z)\left[\sum_{k=1}^{s} \frac{z}{1-\bar{\alpha}_{k} z} \frac{\bar{L}_{1}\left(\alpha_{k}\right)}{\bar{f}\left(\alpha_{k}\right)}+\sum_{k=1}^{t} \frac{z}{1-\bar{\beta}_{k} z} \frac{\bar{L}_{2}\left(\beta_{k}\right)}{\bar{f}\left(\beta_{k}\right)}\right] \\
& \left.-B_{1} z f^{\prime}(z)\left[\sum_{k=1}^{s} \frac{1}{z-\alpha_{k}} \frac{L_{1}\left(\alpha_{k}\right)}{f\left(\alpha_{k}\right)}+\sum_{k=1}^{t} \frac{1}{z-\beta_{k}} \frac{L_{2}\left(\beta_{k}\right)}{f\left(\beta_{k}\right)}\right]\right\} \\
& +O\left(\lambda^{2}\right) .
\end{aligned}
$$

There is a similarity between Equations (2.41) and (2.43) and they may be brought closer together by setting $B_{1}=B b$ in Equation (2.43) and using $b=f\left(\alpha_{k}\right)$ for each index $k$. We state the result as

THEOREM 2.5. Under the conditions and with the notations of Theorem 2.3, $f_{\lambda}(z) \in R(p, q)$ where

$$
\begin{aligned}
& f_{\lambda}(z)=f(z)+\lambda\left\{\frac{B f^{2}(z)}{f(z)-b}-q B f(z)\left[\sum_{k=1}^{s} \frac{L_{1}\left(\alpha_{k}\right)}{\alpha_{k}}+\sum_{k=1}^{t} \frac{L_{2}\left(\beta_{k}\right) b}{\beta_{k} f\left(\beta_{k}\right)}\right]\right. \\
& +\bar{B} z f^{\prime}(z)\left[\sum_{k=1}^{s} \frac{z}{1-\bar{\alpha}_{k} z} \bar{L}_{1}\left(\alpha_{k}\right)+\sum_{k=1}^{t} \frac{z}{1-\bar{\beta}_{k} z} \frac{\bar{L}_{2}\left(\beta_{k}\right) b}{\bar{f}\left(\beta_{k}\right)}\right] \\
& \left.-B z f^{\prime}(z)\left[\sum_{k=1}^{s} \frac{1}{z-\alpha_{k}} L_{1}\left(\alpha_{k}\right)+\sum_{k=1}^{t} \frac{1}{z-\beta_{k}} \frac{L_{2}\left(\beta_{k}\right) b}{f\left(\beta_{k}\right)}\right]\right\} \\
& +O\left(\lambda^{2}\right) \text {. }
\end{aligned}
$$


Further for sufficiently small $\lambda, f(z)$ and $f_{\lambda}(z)$ have in $E$ the same number of zeros and critical points when connted in accordance with their multiplicities.

Equations (2.41) and (2.44) differ only in the terms involving the critical points $\beta_{k}$, but this seems to be an essential difference. If $f(z)$ has no critical points then Theorems 2.4 and 2.5 give the same $f_{\lambda}(z)$. We state this as

THEOREM 2.6. Let $f(z) \in R(p, q)$ and suppose that $f^{\prime}(z) \neq 0$ in $0<|z|<1$. Then with the other conditions, and notations of Theorem $2.2, f_{\lambda}(z) \in R(p, q)$ where

$$
\begin{aligned}
f_{\lambda}(z)= & f(z)+\lambda\left\{\frac{B f^{2}(z)}{f(z)-b}-q B f(z) \sum_{k=1}^{s} \frac{L_{1}\left(\alpha_{k}\right)}{\alpha_{k}}\right. \\
& \left.+\bar{B} z f^{\prime}(z) \sum_{k=1}^{s} \frac{z}{1-\bar{\alpha}_{k} z} \bar{L}_{1}\left(\alpha_{k}\right)-B z f^{\prime}(z) \sum_{k=1}^{s} \frac{1}{z-\alpha_{k}} L_{1}\left(\alpha_{k}\right)\right\} \\
& +O\left(\lambda^{2}\right) .
\end{aligned}
$$

Further for sufficiently small $\lambda, f_{\lambda}^{\prime}(z) \neq 0$, in $0<|z|<1$.

3. Schiffer's formula. In $1943 \mathrm{M}$. Schiffer [13] gave a formula for the variation of a function in the class $R(p, 1)$. In order to facilitate comparison of his result, with that obtained here in $\$ 2$, we alter Schiffer's notation and make a few other minor changes. We use the symbol $X \rightarrow Y$ to show that the symbol $X$ in Schiffer's paper has been changed into $Y$ in the formulas given below. Then $z \rightarrow w, \zeta \rightarrow z, a_{i} \rightarrow b$ and $\rho \rightarrow \lambda b B$, where $\lambda>0$ and $B$ is an arbitrary complex constant. In the general formulation [13, p. 342] $q(w)$ is merely a single-valued function on a certain Riemann surface, but for our purposes we select $q(w)=w /(w-b)$ where $b \neq 0$. Then $f\left(\zeta_{i}\right)=z_{i} \rightarrow f\left(\alpha_{k}\right)=b, k=1,2, \cdots, m$. With these alterations Schiffer's formulas $\left({ }^{2}\right)\left[13\right.$, Equations $(27),\left(27^{\prime}\right)$ and $\left(27^{\prime \prime}\right)$ ] give the following result.

TheOREM 3.1. Let $f(z) \in R(p, 1)$ and let $b$ be a complex number, $b \neq 0$, $b \notin V$ where $V$ is the boundary of the image of $E$ under $f(z)$. Suppose further that the roots $\alpha_{k}, k=1,2, \cdots, s$, of $f(z)=b$ in $E$ are all simple.

Then for $0 \leqq \lambda<\lambda_{0}, f_{\lambda}(z) \in R(p, 1)$ where

$$
\begin{aligned}
f_{\lambda}(z)= & f(z)+\lambda\left\{\frac{B f^{2}(z)}{f(z)-b}-B f(z) \sum_{k=1}^{s} \frac{L_{1}\left(\alpha_{k}\right)}{\alpha_{k}}\right. \\
& +\bar{B} z f^{\prime}(z) \sum_{k=1}^{s} \frac{z}{1-\bar{\alpha}_{k} z} \bar{L}_{1}\left(\alpha_{k}\right)-B z f^{\prime}(z) \sum_{k=1}^{s} \frac{1}{z-\alpha_{k}} L_{1}\left(\alpha_{k}\right) \\
& \left.+i\left(f(z)-z f^{\prime}(z)\right) \Im\left(-B+B \sum_{k=1}^{s} \frac{L_{1}\left(\alpha_{k}\right)}{\alpha_{k}}\right)\right\}+O\left(\lambda^{2}\right),
\end{aligned}
$$

(3) These equations contain misprints. In Equation (27) $q(0)$ should be replaced by $-q(0)$ wherever it appears. This change will induce further changes in Equations (27') and (27'). In our case $q(0)=0$, so the misprints cause no trouble. 
where $B$ is an arbitrary constant and $L_{1}(\alpha)$ is given by Equation (2.36). Further $f_{\lambda}(z)$ and $f(z)$ have in $E$ the same number of zeros, and the same number of critical points when counted in accordance with their multiplicities.

When the last term in Equation (3.1) is dropped, this equation coincides with Equation (2.45) when $q=1$. However in Equation (2.45) it is assumed $f^{\prime}(z) \neq 0$ in $E$, whereas no such assumption has been made in the derivation of Equation (3.1).

We remark that Equations (2.45) and (3.1) should coincide for a univalent function, but actually even in this simple case they differ by the last term in Equation (3.1). In the next few lines we show that this difference can be accounted for by a trivial rotation, and that the last term of Equation (3.1) may be deleted without affecting the validity of Theorem (3.1). Indeed if $f_{\lambda}(z) \in R(p, 1)$ then for any real constant $c, f^{*}(z)=e^{-i \lambda c} f_{\lambda}\left(e^{i \lambda c} z\right) \in R(p, 1)$. Now as $\lambda \rightarrow 0$

$$
\begin{aligned}
f^{*}(z) & =(1-i \lambda c) f_{\lambda}((1+i \lambda c) z)+O\left(\lambda^{2}\right) \\
& =(1-i \lambda c)\left(f_{\lambda}(z)+i c z f_{\lambda}^{\prime}(z) \lambda\right)+O\left(\lambda^{2}\right), \\
f^{*}(z) & =f_{\lambda}(z)+i \lambda c\left(z f_{\lambda}^{\prime}(z)-f_{\lambda}(z)\right)+O\left(\lambda^{2}\right) .
\end{aligned}
$$

If now we apply Equation (3.2) to $f_{\lambda}(z)$ given by Equation (3.1), and set $c=\Im\left(-B+\sum L_{1}\left(\alpha_{k}\right) / \alpha_{k}\right)$ then the last term of Equation (3.1) will drop out.

THEOREM 3.2. Under the conditions and with the notations of Theorem 3.1, $f^{*}(z) \in R(p, 1)$, where

$$
\begin{aligned}
f^{*}(z)= & f(z)+\lambda\left\{\frac{B f^{2}(z)}{f(z)-b}-B f(z) \sum_{k=1}^{s} \frac{L_{1}\left(\alpha_{k}\right)}{\alpha_{k}}\right. \\
& \left.+\bar{B} z f^{\prime}(z) \sum_{k=1}^{s} \frac{z}{1-\bar{\alpha}_{k} z} \bar{L}_{1}\left(\alpha_{k}\right)-B z f^{\prime}(z) \sum_{k=1}^{s} \frac{1}{z-\alpha_{k}} L_{1}\left(\alpha_{k}\right)\right\}+O\left(\lambda^{2}\right) .
\end{aligned}
$$

Further $f(z)$ and $f^{*}(z)$ have in $E$ the same number of zeros and the same number of critical points when counted in accordance with their multiplicities.

4. The Schaeffer-Spencer method. In this section we obtain the generalization of the formula for the variation of a univalent function, given by Schaeffer and Spencer [12, p. 32]. The work is based on two of their lemmas (IV and V) [12, p. 23 and p. 29], which we reproduce below.

Briefly described: a "cut" is made in the complex $z$-plane along an analytic arc and one bank of the cut is then deformed to give a new region $G_{\epsilon}$, and this region is mapped onto the complex $w$-plane "sewing" the cut by a univalent function preserving direction and distance at infinity. Lemma IV gives both an explicit and an approximate form for this function.

Let $\Gamma$ be an analytic Jordan arc which is regular even at its end points $\alpha$ and $\beta$ and let $D$ be a simply-connected region containing $\Gamma$ in its interior. 
Let $P_{\epsilon}(z)$ be regular in $D$ for all complex $\epsilon$ for which $|\epsilon| \leqq \epsilon_{0}$, and satisfy the conditions

$$
\begin{aligned}
\left|P_{\epsilon}(z)\right| & \leqq M, \\
\left|P_{\epsilon^{\prime}}(z)-P_{\epsilon^{\prime \prime}}(z)\right| & \leqq M\left|\epsilon^{\prime}-\epsilon^{\prime \prime}\right|, \\
P_{\epsilon}(\alpha) & =P_{\epsilon}(\beta)=0,
\end{aligned}
$$

for $z$ in $D$. If $\epsilon$ is sufficiently small, then as $z$ moves over $\Gamma$ from $\alpha$ to $\beta$, the point $z+\epsilon P_{\epsilon}(z)$ describes an analytic Jordan $\operatorname{arc} \Gamma_{\epsilon}$ joining $\alpha$ and $\beta$. The two arcs form the complete boundary of an infinite simply-connected region $G_{\epsilon}$, which may be twice covered in a neighborhood of $\Gamma$, such that in moving from $\alpha$ to $\beta$ along $\Gamma$ and from $\beta$ to $\alpha$ along $\Gamma_{\epsilon}$, the area to the left is constantly in the region $G_{\epsilon}$.

Lemma IV. Let $P_{\epsilon}(z)$ satisfy the conditions (4.1) and let $G_{\epsilon}$ be the region defined above. For each sufficiently small $\epsilon$ there is a function $f_{\epsilon}(z)$ which is univalent in $G_{\epsilon}$, and regular there except at $z=\infty$ where it has the development $f_{\epsilon}(z)$ $=z+a_{1}(\epsilon) / z+\cdots$ and $w=f_{\epsilon}(z)$ maps $G_{\epsilon}$ onto the exterior of a Jordan arc in the w-plane in such a way that corresponding points of $\Gamma$ and $\Gamma_{\epsilon}$ are brought together, i.e. for $z$ on $\Gamma$

$$
f_{\epsilon}\left(z+\epsilon P_{\epsilon}(z)\right)=f_{\epsilon}(z) .
$$

Moreover

$$
f_{\epsilon}(z)=z+\frac{\epsilon}{2 \pi i} \int_{\alpha}^{\beta} \frac{P_{\epsilon}(u) f^{\prime}(u)}{f_{\epsilon}(u)-f_{\epsilon}(z)} d u,
$$

and as $\epsilon \rightarrow 0$

$$
f_{\epsilon}(z)=z+\frac{\epsilon}{2 \pi i} \int_{\alpha}^{\beta} \frac{P(u)}{u-z} d u+o(\epsilon)
$$

where $P(u)=P_{0}(u)$ and the $o(\epsilon)$ holds uniformly in $G_{\epsilon}$. The path of integration lies in $D$, but may depend on $z$.

Here $\Gamma$ and $\Gamma_{\epsilon}$ represent the two edges of the cut in the $z$-plane, and Equation (4.2) expresses the fact that the edges are "sewed" together again. In Lemma $\mathrm{V}$, the "cut" and the region $D$ lie in $|z|<1$, and the mapping is now from the "cut" unit circle onto the unit circle, "sewing" the "cut."

Lemma V. Let $\Gamma, D$ and $P_{\epsilon}(z)$ satisfy the conditions of Lemma $I V$, and suppose further that $D$ lies in $|z|<1$, but does not contain the origin. Let $G_{\epsilon}$ be the doubly-connected region bounded by $|z|=1, \Gamma$ and $\Gamma_{\epsilon}$, such that the region lies to the left as $z$ describes $\Gamma$ from $\alpha$ to $\beta$, and $\Gamma_{\epsilon}$ from $\beta$ to $\alpha$. Then there is a function $f_{\epsilon}(z)$ of the form 


$$
w=f_{\epsilon}(z)=\sum_{n=1}^{\infty} a_{n} z^{n}, \quad a_{1}>0,
$$

near $z=0$, univalent and regular in $G_{\epsilon}$, mapping $|z|=1$ onto $|w|=1$, and mapping $G_{\epsilon}$ onto $|w|<1$, minus a Jordan arc, in such a way that corresponding points of $\Gamma$ and $\Gamma$, are brought together, that is Equation (4.2) holds for $z$ on $\Gamma$. Further as $\epsilon \rightarrow 0$

$$
\begin{aligned}
f_{\epsilon}(z)= & z\left\{1+\frac{\epsilon}{2 \pi i} \int_{\alpha}^{\beta} \frac{P(u)}{2 u^{2}} \frac{u+z}{u-z} d u-\frac{\bar{\epsilon}}{2 \pi i} \int_{\alpha}^{\beta} \frac{\bar{P}(u)}{2 \bar{u}^{2}} \frac{1+\bar{u} z}{1-\bar{u} z} d \bar{u}\right\} \\
& +o(\epsilon),
\end{aligned}
$$

where $o(\epsilon)$ holds uniformly in $G_{\epsilon}$. The path of integration depends on $z$ wherever two sheets of $G_{\epsilon}$ overlap.

For the generalization to multivalent functions of the Schaeffer-Spencer formula [12, p. 32], Lemma IV can be used as it stands, but Lemma V requires the following modifications.

Lemma 4.1. For each index $k, k=1,2, \cdots, p$, let $\Gamma^{(k)}$ be an analytic Jordan arc regular even at its end points $\alpha_{k}, \beta_{k}$; let $D_{k}$ be a region containing $\Gamma^{(k)}$; and let $P_{e}^{(k)}(z)$ be regular in $D_{k}$ and satisfy there the conditions (4.1). Suppose further that the regions $D_{k}$ are pairwise disjoint, do not contain $z=0$, and all lie in $|z|<1$. Let $G_{\mathrm{e}}$ be the $(p+1)$-connected region whose complete boundary is the circle $|z|=1$ and the $2 p$ arcs $\Gamma^{(k)}$ and $\Gamma_{e}^{(k)}$ where, for each index $k, \Gamma_{e}^{(k)}$ is the curve described by $z+\epsilon P_{\epsilon}^{(k)}(z)$ as $z$ describes $\Gamma^{(k)}$ and $G_{\epsilon}$ is to the left as $z$ moves on $\Gamma^{(k)}$ from $\alpha_{k}$ to $\beta_{k}$, and as $z$ moves on $\Gamma_{e}^{(k)}$ from $\beta_{k}$ to $\alpha_{k}$. Then there exists a function $f_{\epsilon}(z)$ of the form (4.5) near $z=0$, univalent and regular in $G_{\epsilon}$, mapping $|z|=1$ onto $|w|=1$, and mapping $G_{\text {e onto }}|w|<1$ minus $p$ Jordan arcs, in such a vay that corresponding points of $\Gamma^{(k)}$ and $\Gamma_{e}^{(k)}$ are brought together, that is, for $z$ on $\Gamma^{(k)}$

$$
f_{\epsilon}(z)=f_{t}\left(z+\epsilon P^{(k)}(z)\right), \quad k=1,2, \cdots, p .
$$

Further as $\epsilon \rightarrow 0$

$$
f_{\epsilon}(z)=z\left\{1+\epsilon \sum_{k=1}^{p} S^{(k)}(z)-\bar{\epsilon} \sum_{k=1}^{p} T^{(k)}(z)\right\}+o(\epsilon),
$$

where

$$
\begin{aligned}
& S^{(k)}(z)=\frac{1}{2 \pi i} \int_{\alpha_{k}}^{\beta_{k}} \frac{P^{(k)}(u)}{2 u^{2}} \frac{u+z}{u-z} d u, \\
& T^{(k)}(z)=\frac{1}{2 \pi i} \int_{\alpha_{k}}^{\beta_{k}} \frac{\bar{P}^{(k)}(u)}{2 \bar{u}^{2}} \frac{1+\bar{u} z}{1-\bar{u} z} d \bar{u},
\end{aligned}
$$


and $P^{(k)}(u)=P_{0}^{(k)}(u), k=1,2, \cdots, p$. The error term o( $\left.\epsilon\right)$ holds uniformly in $G_{\epsilon}$, and the path of integration depends on $z$ wherever two sheets of $G_{\epsilon}$ overlap.

Both the existence of $f_{\epsilon}(z)$ and the formula (4.8) follow immediately from Lemma $V$, by induction. First suppose $z_{1}=F_{1}(z)$ is a function "sewing" the first "cut." The existence of this function is assured by Lemma V. Then $F_{1}(z)$ maps the remaining $2 p-2$ analytic arcs again into analytic arcs, and the functions $P_{\epsilon}^{(k)}(z)$ go into new functions $Q_{\epsilon}^{(k)}\left(z_{1}\right)$ defined by the equations

$$
F_{1}(z)+\epsilon Q_{\epsilon}^{(k)}\left(F_{1}(z)\right)=F_{1}\left(z+\epsilon P_{\epsilon}^{(k)}(z)\right), \quad k=2,3, \cdots, p .
$$

Clearly $Q_{\epsilon}^{(k)}\left(z_{1}\right)$ satisfies the conditions of Lemma V in some suitable region in $\left|z_{1}\right|<1$. With a sequence of such mappings, $z_{k}=F_{k}\left(z_{k-1}\right), k=1,2, \cdots, p$, $z_{0}=z$, each mapping "sewing" up one "cut," we can construct the desired function

$$
w=f_{\epsilon}(z)=F_{p}\left(F_{p-1}\left(\cdots\left(F_{1}(z)\right) \cdots\right)\right) .
$$

The formula (4.8) follows immediately from formula (4.6) by induction applied to the function given by (4.12). The proof gives an appearance of being complicated but the incisive fact is that $z_{p-1}=z+O(\epsilon)$ so that in the integrals in (4.9) and (4.10), $z_{p-1}$ may be replaced by $z$ with an error term of $o(\epsilon)$.

We now apply Lemma IV and Lemma 4.1 to obtain a variation formula in the family $R(p, q)$. Let $f(z) \in R(p, q)$, and let $W$ be the Riemann surface onto which $w=f(z)$ maps $|z|<1$. We assume further that there is a simply connected region $D^{*}$ in the $w$-plane such that for each $c$ in $D^{*} f(z)=c$ has precisely $p$ solutions in $|z|<1$. By reducing $D^{*}$ we may assume that $w=0 \notin D^{*}$ and that none of the branch points of $W$ lie over $D^{*}$, so that the inverse function, $z=\phi(w)$, of $f(z)$ can be split into $p$ functions $\phi_{k}(w)$ each singlevalued and regular in $D^{*}$ and mapping $D^{*}$ into regions $D^{(1)}, D^{(2)}, \cdots, D^{(p)}$, satisfying the conditions of Lemma 4.1. Let $\Gamma^{*}$, contained in $D^{*}$, be an analytic Jordan arc, regular even at its end points $\alpha_{k}^{*}, \beta_{k}^{*}$, and let $P_{c}^{*}(w)$ be a regular function in $D^{*}$, satisfying the conditions (4.1), and suppose that $|\epsilon| \leqq \epsilon_{0}$ is so restricted that as $w$ traces $\Gamma^{*}, w+\epsilon P_{\epsilon}^{*}(w)$ traces a curve $\Gamma_{\epsilon}^{*}$ which is in $D^{*}$ for each $\epsilon$. Under these conditions each function $z=\phi_{k}(w)$ maps the curves $\Gamma^{*}$ and $\Gamma_{\epsilon}^{*}$ into curves $\Gamma^{(k)}$ and $\Gamma_{e}^{(k)}$ lying in $D^{(k)}$, and transforms $P_{e}^{*}(w)$ into a function $P_{e}^{(k)}(z)$ defined by the equation

$$
\phi_{k}(w)+\epsilon P_{\epsilon}^{(k)}\left(\phi_{k}(w)\right)=\phi_{k}\left(w+\epsilon P_{\epsilon}^{*}(w)\right), \quad k=1,2, \cdots, p .
$$

From Equation (4.13) it is easy to see that

$$
P_{\epsilon}^{(k)}(z)=P^{*}(f(z)) / f^{\prime}(z)+o(1), \quad k=1,2, \cdots, p .
$$

These regions, curves, and functions satisfy the conditions of Lemma 4.1, 
so that there is a function $z_{1}=g(z)$ of the form (4.8) mapping the "cut" unit circle $|z|<1$ onto $\left|z_{1}\right|<1$ minus $p$ Jordan arcs. If $z=G\left(z_{1}\right)$ is the function inverse to $g(z)$, then

$$
z=G\left(z_{1}\right)=z_{1}\left\{1-\epsilon \sum_{k=1}^{p} S^{(k)}\left(z_{1}\right)+\bar{\epsilon} \sum_{k=1}^{p} T^{(k)}\left(z_{1}\right)\right\}+o(\epsilon) .
$$

We next "cut" $W$ to obtain a new Riemann surface $W_{\epsilon}$. In each of the $p$ sheets lying over the region $D^{*}$, introduce curves $\Gamma^{*(k)}$ and $\Gamma_{e}^{*(k)}, k=1,2, \cdots$, $p$, lying directly over the primitive curves $\Gamma^{*}$ and $\Gamma_{\epsilon}^{*}$. Let $W_{\epsilon}$ be the Riemann surface obtained from $W$ by introducing the $2 p$ new boundary curves $\Gamma^{*(k)}$ and $\Gamma_{\epsilon}^{*(k)}$, where $W_{\epsilon}$ lies to the left as $w$ traces $\Gamma^{*(k)}$ from $\alpha^{*}$ to $\beta^{*}$, and $\Gamma_{\epsilon}^{*(k)}$ from $\beta^{*}$ to $\alpha^{*}$. If now $w_{1}=h(w)$ is the function described in Lemma IV mapping the "cut" $w$-plane onto the $w_{1}$-plane minus a Jordan arc, then this function will take the $p$-sheeted surface $W_{\epsilon}$ into a $p$-sheeted surface minus $p$ Jordan arcs, all lying over the corresponding arc in the $w_{1}$-plane. We note that the new surface will have the same number of branch points, each with the same order of multiplicity as before.

The composite mapping function

$$
f^{*}\left(z_{1}\right)=h\left(f\left(G\left(z_{1}\right)\right)\right)
$$

is regular and $p$-valent in $|z|<1$. To assure that $f^{*}(0)=0$, we subtract the appropriate constant term in Equation (4.4), that is we set

$$
h(w)=w+\frac{\epsilon}{2 \pi i} \int_{\alpha^{*}}^{\beta^{*}} \frac{P^{*}(v)}{v-w} d w-\frac{\epsilon}{2 \pi i} \int_{\alpha^{*}}^{\beta^{*}} \frac{P^{*}(v)}{v} d v+o(\epsilon),
$$

or

$$
h(w)=w\left\{1+\frac{\epsilon}{2 \pi i} \int_{\alpha^{*}}^{\beta^{*}} \frac{P^{*}(v)}{v(v-w)} d v\right\}+o(\epsilon) .
$$

If we return to the $z$-plane, using $v=f(u)$, then

$$
h(f(z))=f(z)\left\{1+\frac{\epsilon}{2 \pi i} \int_{\alpha_{k}}^{\beta_{k}} \frac{P^{*}(f(u)) f^{\prime}(u)}{f(u)(f(u)-f(z))} d u\right\}+o(\epsilon) .
$$

Here any index $k(k=1,2, \cdots, p)$ may be used. Note that $h(f(z))$ has the same number of zeros as $f(z)$ and each zero has the same multiplicity for both functions. From Equation (4.15) we have

$$
f\left(G\left(z_{1}\right)\right)=f\left(z_{1}\right)-z_{1} f^{\prime}\left(z_{1}\right)\left\{\epsilon \sum_{k=1}^{p} S^{(k)}\left(z_{1}\right)-\bar{\epsilon} \sum_{k=1}^{p} T^{(k)}\left(z_{1}\right)\right\}+o(\epsilon) .
$$

Then the function $f^{*}\left(z_{1}\right)$ is easily estimated, using Equations (4.18) and (4.19), we find 


$$
\begin{aligned}
f^{*}\left(z_{1}\right)= & f\left(z_{1}\right)+\epsilon \frac{f\left(z_{1}\right)}{2 \pi i} \int_{\alpha_{k}}^{\beta_{k}} \frac{P^{*}(f(u)) f^{\prime}(u)}{f(u)\left(f(u)-f\left(z_{1}\right)\right)} d u \\
& -z_{1} f^{\prime}\left(z_{1}\right)\left\{\epsilon \sum_{k=1}^{p} S^{(k)}\left(z_{1}\right)-\bar{\epsilon} \sum_{k=1}^{p} T^{(k)}\left(z_{1}\right)\right\}+o(\epsilon) .
\end{aligned}
$$

The coefficient of $z^{q}$ in $f^{*}\left(z_{1}\right)$ is

$$
1+\frac{\epsilon}{2 \pi i} \int_{\alpha_{k}}^{\beta_{k}} \frac{P^{*}(f(u)) f^{\prime}(u)}{f^{2}(u)} d u-\epsilon q \sum_{k=1}^{p} S^{(k)}(0)+\bar{\epsilon} q \sum_{k=1}^{p} T^{(k)}(0)+o(\epsilon) .
$$

To obtain the normalization of Equation (1.1) we divide $f^{*}\left(z_{1}\right)$ by this constant, and we have finally (dropping the subscript on $z_{1}$ )

$$
\begin{aligned}
f_{\epsilon}(z)= & f(z)+\epsilon \frac{f^{2}(z)}{2 \pi i} \int_{\alpha_{k}}^{\beta_{k}} \frac{P^{*}(f(u)) f^{\prime}(u)}{f^{2}(u)(f(u)-f(z))} d u \\
& +\frac{\epsilon}{2 \pi i} \sum_{k=1}^{p} \int_{\alpha_{k}}^{\beta_{k}} \frac{P^{(k)}(u)}{2 u^{2}}\left\{q f(z)-z f^{\prime}(z) \frac{u+z}{u-z}\right\} d u \\
& +\frac{\bar{\epsilon}}{2 \pi i} \sum_{k=1}^{p} \int_{\alpha_{k}}^{\beta_{k}} \frac{\bar{P}^{(k)}(u)}{2 \bar{u}^{2}}\left\{z f^{\prime}(z) \frac{1+\bar{u} z}{1-\bar{u} z}-q f(z)\right\} d \bar{u}+o(\epsilon)
\end{aligned}
$$

is a neighboring $p$-valent function. We have proved the following result.

THEOREM 4.1. Let $f(z) \in R(p, q)$, then the function $f_{\epsilon}(z)$ defined by Equation (4.21) is also in $R(p, q)$ and further $f_{\epsilon}(z)$ has the same number of zeros and critical points as $f(z)$, and each has the same multiplicity. The quantities $P^{(k)}(z)$ are defined by Equations (4.13), (4.14) and $P^{(k)}(z)=\lim _{\epsilon \rightarrow 0} P_{c}^{(k)}(z)$. The limits in the integrals must satisfy the conditions

$$
\begin{aligned}
& f\left(\alpha_{1}\right)=f\left(\alpha_{2}\right)=\cdots=f\left(\alpha_{p}\right), \\
& f\left(\beta_{1}\right)=f\left(\beta_{2}\right)=\cdots=f\left(\beta_{p}\right) .
\end{aligned}
$$

It should be noted that if the region $D^{*}$ initially chosen in the w-plane, is covered by precisely $s$ sheets of $W$, i.e. $c \in D^{*}$ implies $f(z)=c$ has precisely $s$ roots in $|z|<1$, then the sums in (4.21) run from $k=1$ to $k=s$, but the theorem is still valid.

\section{REFERENCES}

1. S. A. Gel'fer, The variation of multivalent functions, Dokl. Akad. Nauk. SSSR vol. 98 (1954) pp. 885-888.

2. - On the coefficient problem for p-valent functions, Dokl. Akad. Nauk. SSSR vol. 106 (1956) pp. 955-958.

3. G. M. Goluzin, Method of variations in conformal mapping I, Mat. Sb. N.S. vol. 19 (61) (1946) pp. 203-236.

4. - Some questions of the theory of univalent functions, Trudy Mat. Inst. Steklov vol. 27 (1949) $111 \mathrm{pp}$. 
5. - Geometric theory of functions of a complex variable, Gosudarstv. Izdat. Tehn.Teor. Lit., Moscow-Leningrad, 540 pp., 1952.

6. A. W. Goodman, Some determinants related to p-valent functions, Trans. Amer. Math. Soc. vol. 63 (1948) pp. 175-192.

7. - On the Schwarz-Christoffel transformation and p-valent functions, Trans. Amer. Math. Soc. vol. 68 (1950) pp. 204-223.

8. A. W. Goodman and M. S. Robertson, A class of multivalent functions, Trans. Amer. Math. Soc. vol. 70 (1951) pp. 127-136.

9. W. K. Hayman, Some applications of the transfinite diameter to the theory of functions, J. Analyse Math. vol. 1 (1951) pp. 155-179.

10. C. Loewner, Untersuchungen über schlichte Konforme Abbildungen des Einheitskreises, I, Math. Ann. vol. 89 (1923) pp. 103-121.

11. M. S. Robertson, Multivalently star-like functions, Duke Math. J. vol. 20 (1953) pp. 539-550. 1950.

12. A. C. Schaeffer and D. C. Spencer, Coefficient regions for schlicht functions, New York.

13. M. Schiffer, Variation of the Green function and theory of the p-valued functions, Amer. J. Math. vol. 65 (1943) pp. 341-360.

The Institute for Advanced Study,

Princeton, N. J.

The University of Kentucky, LEXINGTON, KY. 